\title{
Nachrichten.
}

\section{Biographische Nachricht}

über

Carl Friedrich Bruch, Dr, philos.

grossh. hess. Notar und 1. Director der rheinischen naturforschenden Gesellschaft zu Mainz, Mitglied vieler gelehrten Gesellschaften und Vereine.

(Nach Mittheilungeu der binterbliebenen Angehörigen und Freunde dè Verstorbenen zusammengestellt vom Herausgeber.)

Carl Friedrich Bruch, geboren den 11. März 1789 zu Zweibrücken in der bairischen Pfalz, war den Sohn des dortigen Apothekers Johann Christian Bruch und der jüngere Bruder des bekannten Botanikers und Mitverfassers der Bryologia europaea, Dr. Philipp Bruch daselbst. Der schon durch mehrere Generationen bethätigte naturwissenschaftliche Familienzug führte auch ihn frühe zu selbstständigen Bestrebungen, die von der ersten Knabenzeit an durch sein ganzes Leben, neben einer vielseitigen und anstrengenden amtlichen Wirksamkeit, keinen Augenblick aufgehört haben, seine volle Liebe in Anspruch zu nehmen. Für die Wahl seines engeren Berufs war der frühe Verlust seines Vaters bestimmend, der es ihm unmöglich machte, eine Universität zu besuchen und auf einen bald möglichen Broderwerb hinwies. So kam es, dass er nach dem Austritt aus dem Lyceum zu Mainz, (der damaligen Hauptstadt des Departements vom Donnersberg, zu welchem auch $Z$ weibrücken gehörte) eine Gehülfenstelle bei einem Notar annahm und die im Lyeeum begonnenen naturwissenschaftlichen Studien, welche ihm dort wiederholte Auszeichnungen eingetragen hatten, nur privatim fortsetzen konnte. Er erlangte jedoch eine selbstständige Existenz, als ihm nach der zweiten Einnahme von Mainz durch die Alliirten im Jahre 1814 von der vereinigten österreichisch-preussischen Landesadministration, durch Dekret vom 19. April 1815, ein öffentliches Notariat in Mainz ubertragen wurde, welche Stelle er dann ununterbrochen bis zu seinem freiwilligen Rúcktritte im Jahre 1855 bekleidet hat.

Von dem Vertrauen und der allgemeinen Achtung, welche er sich während seiner vierzigjährigen Dienstzeit erwarb, geben die Ehrenämter Zeugniss, die ihm ausserdem nach und nach von seinen Collegen und Mitbürgern, so wie von der Staatsregierung übertragen wurden. In den langen Friedenszeiten, welche der Besitzergreifung des Grossh. Hess. 
Landestheils auf dem linken Rheinufer am 8. Juli 1816 gefolgt sind, war Bruch nicht nur zu wièderholten malen unter den Wahlmännern seines näheren und weiteren Vaterlandes, sondern es ist auch während dieser Zeit in seiner nunmehrigen Vaterstadt kaum ein gemeinnütziges oder monumentales Wérkirérstanden, zan welchém er nicht als langjähriges Mitglied des Stadtvorstandes und der verschiedenartigsten Vereine thätig mitgewirkt hälte. Wir erwähnen hier nur als allgemeiner bekannte Thatsachen der Entstehung der evangelischen Kirchengemeinde in Mainz, der neuen Casinogesellschaft und des Vereins für Kunst und Literatur, søwie ferner die Errichtung des neuen Theaters, des Gutenbergmonumentes und die vierte Säcularfeier der Erfindung der Buehdruckerkunst im Jahre 1837. Von Seiten der Staatsbehörde wurde er im Jāhre 1838 zum Mitglied der Prüfungskommission für das Justizfach in Rheinhessen und im folgenden Jahre auch des Rekrutirungsrathes

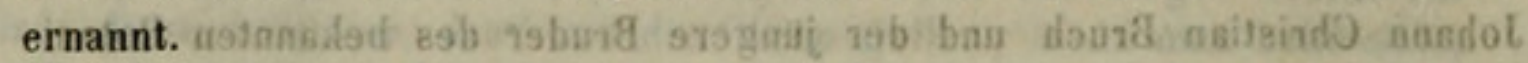

Nur durch eine ungewöhnliche Arbeitskraft und Hingebüng konnte es Bruch möglich werden, neben so zahlreichen und anstrengenden BeA rufsgeschäften eine ornithologische Sammlung anzulegen, welche nach dem Zeugniss, von Kennern schon gegen Ende der zuvanziger Jahre für europäische Vögel, die er/ sämmtlich eigenhändig ausgestopft hatte, vollstândig war. Die sten Abweichungen Ves Alters und Klimas, welche letztere Inamentlich stets seine Aufmerksamkeit erregten. Daneben fühte Bruch eine ausgebreitete ornithologische Correspondenz, schrieb zahlreiche Aufsătze in Okens Isis und gewann Iseiner Lieblingswissenschaft durch glücklich gewählte Vorträge und Demonstrationen Anerkennung in den wissenschaftlichen Kreisen seiner Vaterstadt. Vorzugsweise seinen Bestrebungen und denen der Dr. Dr. Gröser und Gergens verdankt die noch bestehende rheinische naturforschende Gesellschaft zu Mainz, deren/ reichhaltige Sammlungen Bruch als erster Director wesentlich geschaffen hat, im Jahre 1834 ihre Gründung. Was hier nicht planlos, sondern nach Maassgabe der Mittel in wenigen Jahren mit Umsicht geleistet wurde, ist von kompetenten Richtern, namentlich bei Gelegenheit der zwanzigsten Versammlung deutscher Naturforseher und Aerzte zu Mainz im Jahre 1842 , sehr anerkennend ausgesprochen worden *). Der Stadtvorstand hatte zu deren Behuf mit bedeutendem Kostenaufwand das bisher zu mercantilischen Zwecken verwendete ehemalige churfürtliche Schloss neu herstellen und zur Aufnahme sammllicher städtischer Samm- 
lungen, so wie der Sammlungen der rheinischen naturforschenden Gesellschaft, in Stand setzen lassen; und die Versammlung selbst konnte durch die Zahl und die Namen der Theilnehmer als eine der gelungensten betrachtet_werden. Die gesammte Bevölkerung nabm daran den wärmsten Antheil. Bruch als ziveiter Geschäftsführer der Versammlung und gleichzeitiges Mitglied des Stadtvorstandes hatle das Seinige zu dem Erfolge beigetragen.

Ein so glücklich und erfolgreich geführtes Leben sollte leider nicht lange auf seinem Höhepunkte verweilen. Ein Schlaganfall, die unbestrittene Folge zu grosser Anstrengungen in seinem engeren Berufe, der ihn im Jahre 1844 nach kaum vollendetem 55 . Lebensjahre traf, lähmte zwar nicht seinen Eifer, noch auch seine Thätigkeit, zwang ihn aber, sich mit zunehmender/Schwäche und Unbehülflichkeit nach und nach yon allen öffentlichen Geschäften und Ehrenämtern zurückzuziehen. Doch hat er sein Notariat noch volle 12 Jahre nach jenem Unfall verwaltet und die meisten schriftlichen Arbeiten und Correspondenzen, nachdem er mit der linken Hand schreiben gelernit, selbst geführt.

Am schwersten fiel ihm die Uebergabe der geliebten Sammlungen, obgleich er den ersten Schritt dazu that, als noch Niemand daran dachte. Einen Ersalz fand Bruch nur in der Verfolgung speciellerer Studien, $\mathrm{die} /$ ihn viele Jahre beschäftigt hatten und als deren Frucht in dieser Zeitschrift die monographischen Arbeiten über die Gattung Larus Lin. erschienen sind, fo wobei er durch die freundlichen Mittheilungen zahlreicher Fachgenossen, insbesondere aber der Museen $z u$ Berlin and Paris, sowie durch die Sammlung der rheinischen naturforschenden Gesellschaft zu Mainz, welche in dieser Gattung schwerlich ihres gleichen haben idurfte, unterstützt wurde. //Mancherlei schmerzliche Erfahrungen, die Begleiter des Alters, das Dahinsterben von Freunden und Fachgenossen, der Verlust der trefflichen Gattin, ja selbst die furchtbare Explosion vom 18. November 1857, von der auch das Bruch'sche Haus in Mainz hart betroffen und er persönlich gefährdet wurde, erschütterten ihn wohl tief, vermochten aber nicht, seine Lieblingsneigungen zu ersticken, die $i h m$ in allen Zeiten tröstend und erfrischend zur Seite gestanden. Je mehr er sich nach Aussen abschloss und abgeschlossen sah, desto inniger and ausschliesslicher//sehloss er/ sich an das Studium der Natur,//das ihn buchstäblich bis auf sein Sterbebett begleitete. Er starb in Folge eines Grippeanfalles; nach iseinein Krankenlagér von wenigen Tagen, am 21. December $1857 \mathrm{im}$ Kreise der Seinigen, Niemandem befeindet, von Vielen bedauert, von /Allen geachtet! 
B ruch wird von solchen, die ihn in früheren Zeiten gekannt haben, als theilnehmender Freund und liebenswürdiger Gesellschafter geschildert, obgleich seine Stimmung unter dem Einfluss einer reizbaren Constitution leicht durch aüussere Veranlassungen getrübt wurde. "Sie, der nicht immer ganz gesunde und nicht inmer heitere Mann * und ein andermal "launig, witzig, tadelnd und gut, wie ich Sie so gerne habe, " nennt ihn sein vieljähriger Freund Brehm, „gemüthlich, humoristisch" ein Anderer in seinen Briefen aus jener Zeit. Erst in späteren Jahren, namentlich seit dem unseligen Schlaganfall, gewann eine düstere Stimmung die Oberhand, ohne dass er sich in seiner gewohnten Thätigkeit dadurch hätte unterbrechen lassen. Auf seine amtliche Thátigkeit ist, wie auf seinen Privatcharacter, nie der leiseste Tadel gefallen. Er genoss ein unbegränztes Vertrauen, nicht bloss bei nahestehenden Freunden, deren Angelegenheit er in der uneigennützigsten Weise verwaltete, sondern nach allen Seiten und in allen Dingen. Nur diesem Vertrauen in seine Gewissenhaftigkeit und der Leichtigkeit, mit der er arbeitete und jeder neuen Aufgabe gerecht zu werden verstand, verdankte Bruch eine Laufbahn, die ihn von dem unscheinbarsten Anfange zu einer sehr ehrenvollen und in mehrfacher Hinsicht hervorragenden Stellung unter seinen Mitbürgern geführt hat. Es war ihm keineswegs gegeben, sich Bahn zu brechen, wo ihm freiwillige Anerkennung versagt wurde, und Niemand hat sich weniger, als er, um äussere Auszeichnungen beworben. Schen seine vielseitige Amts- und Berufsthätigkeit, obgleich sie ihn in zahlreiche und wichtige Berührungen brachte, würde ihm ein Gesellschaftsleben im gewöhnlichen Sinne nicht gestattet haben; auch wenn ihn nicht eine angeborne Scheu von allen öffentlichen Demonstrationen fern gehalten hätte. Sein innerstes Wesen war Humanität und I.iberalität, aber wie er im Leben keiner sogenannten Parthei weder in kirchlichen noch in politischen Dingen angehörte, suchte er auch die Verwirklichung des Humanismus nicht auf äusserem Wege mit künstlichen Mitteln, sondern als ächter Naturforscher, der den Gründen der Erscheinung nachgeht und ihre natürliche Entwicklung voraussieht, auf dem unscheinbareren, aber sicheren Wege der allgemeinen Bildung, die ihm stets als letztes Ziel vorschwebte und für welche er auch in seinem Kreise nach seinen Kräften redlich gewirkt hat. Nichts konnte ihn so sehr bis zur Leidenschaftlichkeit erregen, als lügnerisches Wesen, geschraubte Unnatur und Grossthuerei; Nichts war ihm mehr zuwider, als lärmende Rohheit und Unmässigkeit. Einer der hervorstechendsten Characterzüge war seine Anhänglichkeit an Familienbande und Familienfesté, insbesondere die Liebe zu seinen Kindern, für deren Ausbildung er 
dreifach opferte, was ihm selbst in der Jugend versagt war. Er hatte buchstäblich keinen Feind und keinen Neider, obgleich seine äusseren Verhältnisse, die blos die Ergebnisse seiner angestrengten Thätigkeit waren, für günstige galten. Selbst seine oft allzugrosse Empfindlichkeit und Delikatesse in Geschäftsangelegenheiten, obgleich sie den Verkehr mit ihm erschwerte, vermehrte nur das allgemeine Vertrauen. So kann man in Wahrheit von ihm sagen, dass er das seltene Glück gehabt, trotz schwerer Prüfungen alle seine Lebenszwecke erfüllt zu sehen und, indem er stets sich selber opferte, alle Umgebenden von ihm befriedigt und von jeder Seite seiner Thätigkeit eine ehrenvolle Spur zu hinterlassen.

Von B ru ch s schriftstellerischer Thätigkeit zeugen folgende Schriften und Aufsätze:

1. Amtlicher Bericht über die 20. Versammlung deutscher Naturforscher und Aerzte zu Mainz im September 1842. Herausgegeben von den Geschäftsfuhrern, Med. Rath Dr. Gröser und Notar Bruch. Mit 2 Steindrucktafeln. Mainz. 1843. 4.

2. Verzeichniss der in dem ehemaligen Kurfürstlichen Schlosse zu Mainz aufgestellten Sammlungen der rheinischen naturf. Gesellschaft. Erste Abtheilung. Säugethiere und deren Scelette. Mainz 1843. 4.

3. Zoologische Bemerkungen (über Mäuse und Falken). Isis von Oken. 1824. S. 674.

4. Ornithologische Bemerkungen (Haare im Kukuksmagen u. a.). Ebenda 1825 . S. 577.

5. Ornithologische Beiträge (besonders über Subspecies). Ebenda 1828. S. 718.

6. Ueber einige Artkennzeichen der Vögel (Zahl der Steuerfedern, Lănge der Schwungfedern u a.). Ebenda 1829. S. 630.

7. Beitrag zur Beschreibung des Geieradlers (Farbe der Iris). Ebenda 1831. S. 404 .

8. Einige Bemerkungen über Aufblühen und Verwelken, Entstehen und Verschwinden in der Natur. Ebenda 1843. S. 425.

9. Monographische Uebersicht der Gattung Larus Lin. Journal für Ornithologie. I. S. 46.

10. Vermischtes über Vögel in der Umgegend von Mainz. Bd. II. S. 276 .

11. Revision der Gattung Larus. Ebenda Bd. III. S. 273. (Hervorgerufen durch die Bemerkungen vom Prinzen Bonaparte im Magazin de Zoologie. 185t. Nr. 11.) 
Von wissenschaftichen Vorträgen, die Bruch zu verschiedenen Zeiten gehalten, enthält das Vereinsblatt des Vereins für Kunst iund Literatur zu Mainz, dessen vieljähriges Vorstandsmitglied und Präsident (im Jahre 183џ) B ruch war, folgende Auszüge und vollständige Abdrücke: 119:4 12. Am 26. August 1825. Ueber die Eidertauchente.

ans) 13. Am 16. Jan 1826. Ueber Ornithologie, (insbesondere über ihre ästhetische Bedeutung).

14. Am 20. Febr. 1826. Ueber die Singwerkzeuge der Vögel mit Vorweisung von Präparaten.

15. Am 29. Jan. 1827. Ueber das Einhorn und die Einhornsage (historisch und kritisch, vollständiger Abdruck).

16. Am 7. Dez. 1827. Ueber die Naturgeschichte, besonders die Verwandlung des Frosches.

17. Am 11. Jan. 1828. Ueber nordische Seevögel.

18. Am 19. Dez 1828. Ueber fossile Knochen, die bei Nierstein im Rhein, gefunden wurden.

19. Am 11. Dez 1829. Bericht über die Naturforscherversammlung in Heidelberg.

us 20. Am 5. Nov. 1830. Ueber die Entwicklungsgeschichte der Thierwelt und die neueren naturphilosophischen Theorien darüber.

Ausserdem finden/sich in seinem Nachlasse die Concepte von folgenden ungedruckten Vorträgen, die er in den Versammlungen und bei den Stiftungsfesten der rheinischen naturf. Gesellschaft gehalten hat.

21. Ueber die Wanderungen der Thiere.

22. Ueber die Wohnungen der Thiere.

23. Ueber die Hausthiere.

24. Ueber den Begriff und die Entwicklung der Nafurgeschichte und der Zuologie insbesondere.

25. Classification der Säugethiere.

26. Classification der Vögel.

27. Ueber die Raubthiere.

28. Ueber die bei uns gebräuchlichen und angeordneten Verfolgungen mancher als schädlich bezeichneten/Thiere.

29. Ueber den Kranich.

30. Ueber den Kukuk.

31. Ueber die erloschenen und erlöschenden Thiere, veranlasst durch die Entdeckung eines Fossilienlagers zu Weichenau bei Mainz im Jahre 1840. 


\section{$2 \mathrm{BHL}$ Biodiversity Heritage Library}

1858. "Biographische Nachricht." Journal fu

r Ornithologie 6, 331-336. https://doi.org/10.1007/bf02004725.

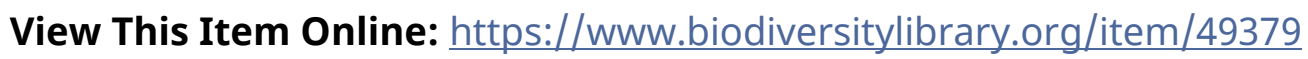

DOI: https://doi.org/10.1007/bf02004725

Permalink: https://www.biodiversitylibrary.org/partpdf/141366

\section{Holding Institution}

Natural History Museum Library, London

\section{Sponsored by}

Natural History Museum Library, London

\section{Copyright \& Reuse}

Copyright Status: Public domain. The BHL considers that this work is no longer under copyright protection.

This document was created from content at the Biodiversity Heritage Library, the world's largest open access digital library for biodiversity literature and archives. Visit BHL at https://www.biodiversitylibrary.org. 